\title{
Accelerated aging mechanisms in chronic obstructive pulmonary disease
}

\author{
Margarida Pimenta Valério ${ }^{1}$, Pedro Carvalho Sá ${ }^{2}$, Denny Marques Rodrigues ${ }^{1}$ and Catarina Ferreira ${ }^{3}$ \\ ${ }^{1}$ Pulmonology Resident; Serviço de Pneumologia do Centro Hospitalar e Universitário de Coimbra, Coimbra, Portugal \\ ${ }^{2}$ Physical Medicine and Rehabilitation Resident; Centro de Medicina de Reabilitação da Região Centro - Rovisco Pais, Tocha, Portugal \\ ${ }^{3}$ Pulmonology Consultant; Serviço de Pneumologia do Centro Hospitalar e Universitário de Coimbra, Coimbra, Portugal
}

\begin{abstract}
Chronic obstructive pulmonary disease (COPD) is projected to become the third leading cause of death worldwide in 2030 . It is a chronic disease that is mostly present in the elderly. A lot of COPD associated features (anatomical and functional) are part of normal lung aging that seems to be accelerated in this disease. LópezOtín, et al. have proposed nine cellular and molecular hallmarks of aging. These hallmarks seem to be present in COPD suggesting that accelerated aging could be a part of the pathogenesis of this disease. Therefore, new potential therapeutic targets based on these mechanisms could modify the evolution of this disease providing better quality of live and prolonged life expectancy.
\end{abstract}

\section{Introduction}

Aging is characterized by progressive deterioration of physiological integrity that occurs after the reproductive phase of life is complete, leading to impaired function and increased vulnerability to death [1]. The study of biological aging processes has developed rapidly throughout the last years and a lot of research has been made to analyse the presence of these mechanisms of accelerated aging in degenerative chronic diseases - ischaemic heart disease, diabetes, Alzheimer's disease and also chronic obstructive pulmonary disease (COPD) [2]. COPD is projected to become the third leading cause of death worldwide in 2030. It is a disease that develops gradually. Most exposures such as cigarette smoking, biomass smoke, and occupational exposures take decades to develop and to induce COPD, hence the vast majority of patients are elderly [1]. Also a lot of COPD-associated features (including airflow limitation and loss of parenchymal tissue) are part of normal lung aging and smoking has been considered an age accelerating factor [3]. LópezOtín, et al. have proposed nine cellular and molecular hallmarks of aging [4]. Therefore, the purpose of this review is to discuss the current evidence for the involvement of aging hallmarks in the pathological features of COPD and how this information can create new potential targets for treatment of this disease.

\section{Methods}

A research was developed for the purpose of this review - pubmed database search of articles published in the last five years ("lung aging copd"). Articles that reflected studies/reviews of aging mechanisms in COPD were selected. We used López-Otín, et al. article in the hallmarks of aging to guide this review.

\section{Discussion}

\section{Physiological changes of the lung with aging}

As part of the normal aging process several physiological changes occur in the lung. After the age of 20-25 years lung function declines as consequence of structural and physiological changes in the lung [2,5], resulting in increased risk of breathlessness and increased prevalence of chronic pulmonary disease in the elderly [6]. In normal healthy subjects, there is an annual decrease in forced expiratory volume in the first second (FEV1) of approximately $20 \mathrm{ml} /$ year in those aged 25-39 and up to $38 \mathrm{ml} /$ year in those 65 years old [1]. In COPD there seems to be an acceleration in FEV1's decline but recent studies show that there is marked individual variability in the decline of FEV1 and that COPD might also be a consequence of suboptimal lung growth in childhood [6]. In addition to changes in FEV1 there is a decrease in the static elastic recoil of the aging lung. This happens because of progressive dilation of the alveolar ducts along with loss of supporting tissues of the peripheral airways - the so called "senile emphysema". This type of emphysema has a more homogenous airspace dilation and there is no alveolar wall destruction as opposed to emphysema related to COPD [1]. In the aging process there is also a decrease in the compliance and of the chest wall and respiratory muscle strength. All these changes result in increase of the residual volume and decrease in vital capacity [2].

\section{Lung aging and COPD phenotypes}

There are different well-known COPD phenotypes and lung aging seems to be more involved in some of these phenotypes than others. The aging lung is inclined to develop an emphysema-like phenotype. The frequent exacerbator phenotype is also important, as exacerbations are in general linked to infections and susceptibility for infections increases with age. Finally, patients with severe early-onset COPD have

*Correspondence to: Margarida Pimenta Valério, Pulmonology Resident; Serviço de Pneumologia do Centro Hospitalar e Universitário de Coimbra, Coimbra, Portugal

Received: December 07, 2020; Accepted: December 11, 2020; Published: December 14, 2020 
a severe disease at a relatively young age, so if accelerated aging is an important contributor to COPD pathology, it should be more clear in these patients [2].

\section{Aging hallmarks in COPD}

As referred previously the hallmarks of aging were summarised in a review by López-Otín, et al. An overview of these hallmarks in lung aging and lung disease was posteriorly developed in several studies. The aging hallmarks were divided in this review into primary hallmarks (causes of damage) - genomic instability, telomere attrition, epigenetic alterations and loss of proteostasis - antagonistic hallmarks (responses do damage) - deregulated nutrient-sensing, mitochondrial disfunction and cellular senescence - and integrative hallmarks (culprits of the phenotype) - stem cell exhaustion and altered intercellular communication [4].

\section{Genomic instability}

Aging has been considered to result from accumulation of genetic damages and defects in DNA repair. Several markers to these features have also been demonstrated in COPD lungs and may contribute to the pathological processes. Oxidative stress is recognized as a mechanism for DNA damage of premature aging and is known to occur in patients with COPD. This was verified by the presence of biomarkers of oxidative stress and of oxidative damage to DNA in the lungs of these patients [6]. The DNA damage marker gamma-H2A histone family member $\mathrm{X}(\gamma$-H2A.X) is increased in alveolar walls, including type I and type II epithelial cells, endothelial cells and in small airways of patients with COPD [2]. There is also evidence of failure in DNA strand break repair in COPD [6]. The anti-aging protein sirtuin 6 (SIRT6) which is considered to be protective against DNA damage and senescence is decreased in lung tissue homogenates from COPD patients with corresponding elevated levels of $\gamma$-H2A.X marker [2]. Also, Ku70 another DNA repair marker - is decreased in leucocytes derived from COPD patients and its expression is negatively correlated with age [2].

Nuclear laminins constitute the major components of the nuclear lamina and regulate genomic stability [4]. Downregulation of laminin $\mathrm{B} 1$ has been recognized as a crucial step for the development of full senescence. A recent study also suggests that laminin B1 reduction is involved in the progression of cellular senescence during COPD pathogenesis through aberrant mammalian target of rapamycin (mTOR) signaling [7].

\section{Telomere shortening}

Telomeres are the terminal part of each DNA strand and keep the integrity of the chromosome. After each cell replication cycle or in response to stressors their length shortens, therefore it is considered as a biomarker of biological age. Once telomeres reach a critical size the DNA damage response is unleashed, which leads to cell cycle arrest and senescence of cells [8].

There is evidence that COPD is associated with shortened telomeres. Reduced telomeres length in circulating leukocytes of patients with COPD has been demonstrated in several studies [2]. This difference was verified in comparison with non-smoker and smoker controls and was irrespective of age, sex, spirometry values, Body Mass Index (BMI) and history of cigarette smoking [9]. Also, a meta-analyses of 14 studies showed a positive association between spirometric indices of airflow limitation and leucocyte telomere length [6]. In a longitudinal study (BODE cohort) telomere shortening in peripheral blood cells was accelerated in COPD patients, and its rate of attrition seemed to depend on baseline telomere length, although these findings did not correlate with lung function decline [10]. However, another study showed that only the heavy smoker patients with shorter telomeres at baseline had accelerated lung function decline, suggesting that telomere attrition might increase individuals susceptibility to the effects of cigarette smoking [8]. One study revealed that telomeres length of alveolar type II cells and pulmonary vascular endothelial cells from COPD patients are significantly decreased compared with those of non-smokers [8]. Despite this, another study of telomeres in airway epithelial cells suggested that telomere associated DNA-damage foci (TAF) is a more robust marker of senescence in COPD than telomere length [11]. The levels of telomere protection protein 1 (TPP1) were also evaluated and were reduced in lung homogenates from COPD patients compared to non-smoking controls [2]. There are also studies in knockout mice that suggest that if there is a telomerase (protective telomere enzyme) dysfunction/deficiency, posteriorly, it leads to premature aging with COPD-like features including dilation of alveolar sacs and fibrosis [8].

\section{Epigenetic alterations}

A range of epigenetic alterations caused by DNA methylation, histone modification and non-coding RNAs, are considered a hallmark of aging, resulting in chromatin remodeling and alteration of gene expression [6].

It has been postulated that DNA methylation status of particular CpG sites ("epigenetic clock"), can be used in an algorithm to predict biological age [2]. Altered DNA methylation induced by cigarette and wood smoke exposure has been related to COPD pathogenesis and it seems to persist even after smoking cessation [8]. Differential DNA methylation has been detected in lymphocytes from patients with COPD compared with healthy control subjects and seems to be correlated with the severity of the disease [6]. DNA isolated from small airways of COPD patients also showed aberrant DNA methylation affecting hundreds of genes, which results in altered expression of genes involved, for instance, in small airway remodeling [1]. A recent study recognized RNA expression and DNA methylation level of agingrelated genes to be changed in COPD males compared to the control group. The identified GpC markers in this study were also associated with the severity of COPD [12].

Histone deacetylases (HDAC) can reduce the acetylation of histones. HDAC activity is reduced in peripheral lung tissue, alveolar macrophages and bronchial biopsies of COPD patients compared to controls and its associated with disease severity [2]. It causes an increase in acetylation of histones in the promoter region of pro-inflammatory genes, resulting in chronic inflammation [1]. Mercado, et al. showed that downregulation of HDAC2 causes nuclear erythroid-related factor 2 (Nrf) instability, leading to the decrease synthesis of anti-oxidant molecules, which increases the susceptibility of cells to oxidative stress related to cigarette smoke (CS) exposure [8]. Another important reference is that corticosteroids reduce inflammation by recruiting HDAC2 to silence the activated NF-kB-stimulated inflammatory gene complex. Reduction of HDAC2 activity in COPD may account of the corticosteroid insensitivity [1].

Sirtuins are type III HDACs and have been studied as potential antiaging factors. They act on histone residues in DNA that are essential to maintenance of silent chromatin during histone deacetylation [6]. Of seven known sirtuins, SIRT1, SIRT3 and SIRT6 are extensively studied in the aging process. Reduction of SIRT1 promotes acetylation of targeted proteins including FOXO3, p53, MMP9 and RelA/p65 thereby enhancing autophagy, cellular senescence, fibrosis and inflammation 
[13]. In a study with mice, silencing SIRT1 causes airspace enlargement, decreased lung function and reduced exercise tolerance [8]. SIRT3 plays a major role in inhibiting ROS in mitochondria [8]. SIRT6 promotes degradation of $\mathrm{p} 21$, which inhibits senescence, and prevents fibrosis by suppressing IL-1 $\beta$ secretion. SIRT6 also inhibits cellular senescence by inducing autophagy. So, even tough the mechanism of autophagy regulation between SIRT1 and SIRT6 are opposing, reduction in both sirtuins appears to be associated with COPD development [13].

\section{Loss of proteostasis}

Proteostasis refers to the systems in place to help maintain functional proteins in the cell. This involves stabilization of correctly folded proteins and degradation of misfolded proteins. This is performed by chaperones such as heat shock proteins (HSP) and proteolytic systems such as the ubiquitin-proteossome and the lysosome-autophagy system - they function to prevent accumulation of damage proteins [1]. HSPs are strongly upregulated in aging to preserve proteostasis and this happens as well to several HSPs in COPD [1]. Aging cells contain more damaged proteins. This accumulation of proteins also happens in COPD lungs [2]. Accumulation of ubiquitinated proteins and the de-ubiquitinating enzyme and aggregation marker ubiquitin C-terminal hydrolase L1 (UCH-L1) is increased in the lung tissue of patients with COPD and correlates with the loss of function $[2,6]$. CS causes oxidative stress which leads to accumulation of misfolded proteins in the endoplasmic reticulum and it also reduces proteossomal activity in the alveolar epithelial cells [8]. Prolonged accumulation of dysfunctional proteins eventually causes apoptosis and triggers chronic inflammation, probably contributing to the pathogenesis of COPD [1].

Autophagy has a protective role as a response to exogenous stress. However, prolonged and excessive autophagy has been associated with cell death [6]. There is emerging evidence that decreased autophagy causes cellular senescence and plays a vital role in the pathogenesis and progression of COPD [14]. As mentioned before SIRT6 prevents cellular senescence by promoting autophagy activation - and CS exposure decreases SIRT6 in human bronchial epithelial cells [14]. CS-exposed bronchial epithelial cells and lung tissue of CODP patients demonstrate insufficient autophagic clearance [15]. The total number of aggresome bodies are increased in the lungs of COPD patients and smokers compared to those of non-smokers and it seems to be related to the severity of the emphysema and alveolar senescence [8]. There is increasing evidence that the autophagy pathway may be a biomarker to diagnose early COPD and also a viable therapeutic target.

\section{Deregulated nutrient-sensing}

Nutrient-sensing is the cell's ability to recognize and respond to fuel sources such as glucose. With aging, cells lose their ability to sense and respond to these changes. A crucial kinase, mTOR, has the ability to sense a variety of nutrients and to alter cellular metabolism. Inhibition of mTOR by rapamycin has proven to extend lifespan in mammals [1]. There is other important pathway which is insulinlike growth factor (IGF) signalling. The mTOR pathway responds to nutrient excess (IGF-1 signalling) as well as to caloric restriction (AMPK and FOXO pathway). With increasing in age mTOR-IGF signalling is upregulated whereas AMKP/FOXO is downregulated (the decrease in nutrient signalling with caloric restriction can induce longevity) [8]. The activity of PI3K/AKT/mTOR is increased in total lung tissue and leukocytes of COPD patients [5]. In addition SIRT6 is considered to attenuate the IGF-mTOR pathway. IGF1 protein levels also seem to be elevated in airway epithelial cells of patients with chronic bronchitis [2]. Prophylactic administration of an AMKP activator, metformin, reduced elastase-induced airspace enlargement, inflammatory responses and cellular senescence in mice [8]. The levels of FOXO3 are significantly reduced in lungs of smokers and patients with COPD and reduction of FOXO3 in CS-exposed mice led to pulmonary emphysema, aggravated inflammatory response and downregulation of antioxidant genes [6].

\section{Mitochondrial dysfunction}

Mitochondria regulates oxidative stress and cellular homeostasis [6]. With aging mitophagy that clears damaged mitochondria may become dysfunctional leading to unwanted mutations in mitochondrial DNA (mtDNA), accumulation of damaged mitochondria and a buildup of reactive oxygen species (ROS). Dysfunctional mitochondria induce cellular senescence [8]. PINK1-PARK2 mediated mitophagy regulates mitochondrial ROS and cell death during COPD pathogenesis [16]. Mitochondrial fission is predominant in diseased cells where the elimination of damaged mitochondria occurs via mitophagy. In bronchial epithelial cells from patients with COPD mitochondrial appear fragmented [5]. The structural changes of mitochondria seem persistent even after depletion of cigarette smoking [8]. Elevated levels of oxidative stress and reduction of mitochondrial numbers are observed in COPD lungs $[2,6]$. Also mitochondrial dysfunction has been well described in skeletal (including respiratory) muscles of COPD patients [1]. In airway smooth muscle mitochondrial dysfunction is present and can be reversed with the mitochondria-targeted antioxidant MitoQ that reduces inflammation and airway responsiveness [8]. Finally, a study by Even, et al. shows that heme-oxigenase-1 (HO-1) induction protects against mitochondrial dysfunction by restoring mitophagy and attenuating senescence in COPD fibroblasts [17].

\section{Cellular senescence}

Cellular senescence is a cell state in which cells stop dividing as a mechanism to prevent tumorigenesis and tissue damage. Senescent cells can be cleared by the immune system, but upon aging the number of senescent cells increases in tissues [2]. Senescent cells, unlike apoptotic cells, remain metabolically active and exhibit the senescence-associated secretory phenotype (SASP) that leads to cellular dysfunction, failure to repair (increased secretion of metalloproteinases (MMP) and growth factors), impaired immunity and chronic, low grade, lung inflammation $[6,18]$. In the blood of COPD patients, biomarkers of the SASP response (IL-1, IL-6, chemokine ligand 8, tumour necrosis factor (TNF)- $\alpha$, transforming growth factor (TGF)- $\beta$, MMP2 and MMP-9) are increased [8]. The senescent cells in general demonstrate more DNA damage and shorter telomeres. These changes have been noted in various lung tissue of COPD and control subjects including lung fibroblasts, airways epithelial cells, alveolar epithelial cells and pulmonary vascular epithelial cells [8]. Cellular senescence involves the activation of the tumour suppression pathways (p53, p21 and the p16 $6^{\mathrm{INK} 4 \mathrm{a}}$ ) [19]. The p16 pathway was found to be increased in total lung tissue, alveolar cells, airway epithelial cells, smooth muscles, endothelial cells and fibroblasts of COPD patients. Similarly the presence of p21 was increased in total lung tissue, alveolar cells, smooth muscle cells and leucocytes of COPD patients [2]. Lung macrophages of patients with COPD also express the senescence marker p21 [19]. Moreover the percentages of p16 and p21-positive cells (alveolar type II and endothelial cells) were negatively correlated with FEV1\%pred [2]. Despite these reports, in an animal model of COPD/emphysema p16 deletion did not protect against airspace enlargement and decline in lung function [20]. 


\section{Stem cell exhaustion}

Adult tissue is thought to reside in a quiescent state. Upon injury, (stem) cells can be activated and are able to proliferate and (trans) differentiate into other cell types, according to their plasticity [2]. Many different types of progenitor cells are seen in the lung. Basal cells found in the airway epithelium can self-renew and differentiate into ciliated and secretory cells; club cells in the bronchioles can give rise to ciliated and secretory cells and in the distal lung; alveolar type II epithelial cells can give rise to alveolar type I cells and function as progenitor cells [1]. In COPD, basal progenitor cells that are necessary for airway epithelial differentiation have a reduced regenerative capacity [6]. Progenitor cell depletion has been shown to correlate with severity of lung function impairment. Endothelial progenitor cells (EPCs) of smokers and COPD patients demonstrate cellular senescence and DNA damage compared to those isolated from non-smokers. Also, circulating hematopoietic progenitors with the ability for tissue repair and EPCs are reduced by up to $30-50 \%$ in COPD patients and correlate with exercise capacity and severity of airflow limitation [8].

\section{Altered intercellular communication}

Aging also leads to changes in intercellular communication including endocrine, neuroendocrine and neuronal signaling. For instance, there is a circulating protein growth differentiator factor 11 (GDF11) which delays aging in mice and whose levels are decreased in COPD patients [8]. Also, inflammation is one way cells communicate with each other [1]. A prominent aging-associated alteration in intercellular communication is "inflammaging" [4]. Chronic low grade inflammation associated with aging occurs due to an increase in secretion of proinflammatory cytokines by senescent cells and progressive dysfunction of the immune system, which fails to clear pathogens and senescent cells - immunosenescence [1]. Aging is associated with decreased epithelial barrier function, abnormalities in both cilia structure and function, and reduced production of antimicrobial and anti-inflammatory peptides produced by epithelial cells [21]. Several recent studies have investigated how aging might affect immune dysregulation in COPD. For instance, a study demonstrated that ageinduced lung inflammation is further elevated after CS exposure in old mice, potentially via an age-induced change in immune susceptibility to CS thereby accelerating the pathophysiological changes in COPD [22]. In this context, from the COPD standpoint, chronic inflammation can develop when inflammation-prone aging lungs are repeatedly exposed to respiratory irritants, such as CS, ultimately leading to tissue destruction and the development of COPD [21]. Other studies observed that Klotho, an anti-aging protein with anti-inflammatory properties, is reduced in alveolar macrophages and airway epithelial cells of patients with COPD [2]. Also, intrinsically abnormal senescent immune cells, accumulation of damage-associated molecular patterns (DAMPs) from the dying senescent cells in the setting of decreased autophagy in aging cells, or SASP produced by senescent cells, can also contribute to the development of inflammation in the lungs of smokers, possibly leading to COPD [21]. Furthermore, decreased expression of MiR$125 \mathrm{a}$ and $\mathrm{b}$ in COPD has been linked to inflammaging and impaired immune response. MiR-125a reduction resulted in NF- $\kappa B$ activation, with induction of pro-inflammatory cytokines, while in parallel low levels of miR-125a and b supress viral clearance [2]. Also, club cell protein 16 deficiency leads to an accelerated lung aging phenotype in mice, which is characterized by emphysema and increased pulmonary inflammation through the activation of NF- $\kappa \mathrm{B}$ [8]. This data suggests that inflammaging and immunosenescence can have a critical role in the development of COPD. Therefore, by regulating these factors we may slow the progression of the disease.

\section{Therapeutic implications}

Antiaging therapies that target the accelerated aging process hold promise for the long-term treatment of aging-related diseases. The treatment of COPD may be administered through inhalation devices to reduce systemic side effects that might be otherwise dose limiting [5]. Potential therapeutic targets include modulation of the mTOR, FOXO and AMPK pathways, specific anti-aging molecules such as sirtuins, Klotho and GDF11, infusion of "young serum", epigenetic modified proteins, or anti-oxidants such as Nrf2 activators and HSP inhibitors [8]. To date, there is no convincing evidence of the beneficial effects in COPD of any of the already studied anti-aging drugs.

\section{Conclusion}

The aging process has been extensively studied in the last years and COPD as other age related diseases (atherosclerosis, hypertension, type 2 diabetes, metabolic syndrome, cancer, etc) shares similar molecular and cellular changes (aging hallmarks). We must focus our efforts on new research to help distinguish the normal aging of the lung and the aging lung in COPD. This will allow us to identify modifiable aging targets responsible for the pathogenesis of COPD, therefore giving us the possibility to delay the natural history of this disease.

\section{References}

1. Kukrety SP, Parekh JD, Bailey KL (2018) Chronic obstructive pulmonary disease and the hallmarks of aging. Lung India 35: 321-327. [Crossref]

2. Brandsma CA, de Vries M, Costa R, Woldhuis RR, Königshoff M, et al. (2017) Lung ageing and COPD: is there a role for ageing in abnormal tissue repair? Eur Respir Rev 26: 170073. [Crossref]

3. Rutten EP, Gopal P, Wouters EF, Franssen FM, Hageman GJ, et al. (2016) Various mechanistic pathways representing the aging process are altered in COPD. Chest 149 : 53-61. [Crossref]

4. López-Otín C, Blasco MA, Partridge L, Serrano M, Kroemer G, et al. (2013) The Hallmarks of Aging. Cell 153: 1194-1217. [Crossref]

5. Mercado N, Ito K, Barnes PJ (2015) Accelerated ageing of the lung in COPD: new concepts. Thorax 70: 482-489. [Crossref]

6. MacNee W (2016) Is chronic obstructive pulmonary disease an accelerated aging disease? Ann Am Thorac Soc 2016; 13: 429-437. [Crossref]

7. Saito N, Araya J, Ito S, Tsubouchi K, Minagawa S, et al. (2019) Involvement of laminin B1 reduction in accelerated cellular senescence during chronic obstructive pulmonary disease pathogenesis. J Immunol 202:1428-1440.

8. Yoon YS, Jin M, Sin DD (2019) Accelerated lung aging and chronic obstructive pulmonary disease. Expert Rev Respir Med 13: 369-380. [Crossref]

9. Sadr M, Mugahi SM, Hassanzadeh G, Nadji SA, Kiani A, et al. (2015) Telomere shortening in blood leukocytes of patients with chronic obstructive pulmonary disease. Tanaffos 14: 10-16. [Crossref]

10. Cordoba-Lanus E, Cazorla-Rivero S, Espinoza-Jimenez A, de-Torres JP, Pajares MJ, et al. (2017) Telomere shortening and accelerated aging in COPD: findings from the BODE cohort. Respir Res 18: 59. [Crossref]

11. Birch J, Anderson RK, Correia-Melo C, Jurk D, Hewitt G, et al. (2015) DNA damage response at telomeres contributes to lung aging and chronic obstructive pulmonary diseases. Am J Physiol Lung Cell Mol Physiol 309: 1124-1137. [Crossref]

12. Du X, Yuan L, Wu M, Men M, He R, et al. (2019) Variable DNA methylation of agingrelated genes is associated with male COPD. Respir Res 20: 243. [Crossref]

13. Chun P (2015) Role of sirtuins in chronic obstructive pulmonary disease. Arch Pharm Res 38: 1-10. [Crossref]

14. Kuwano K, Araya J, Hara H, Minagawa S, Takasaka N, et al. (2016) Cellular senescence and autophagy in the pathogenesis of chronic obstructive pulmonary disease (COPD) and idiopathic pulmonary fibrosis (IPF). Respir Investig 54: 397-406. [Crossref] 
15. Vij N, Chandramani SP, Van Westphal C, Hole R, Bodas M, et al. (2018) Cigarette smoke-induced autophagy impairment accelerates lung aging, COPD-emphysema exacerbations and pathogenesis. Am J Physiol Cell Physiol 314: C73-C87. [Crossref]

16. Tsubouchi K, Araya J, Kuwano K (2018) PINK1-PARK2-mediated mitophagy in COPD and IPF pathogenesis. Inflamm Regen 38:1-9. [Crossref]

17. Even B, Fayad-Kobeissi S, Gagliolo JM, Motterlini R, Boczkowski J, et al. (2018) Heme-oxygenase-1 induction attenuates senescence in chronic obstructive pulmonary disease lung fibroblasts by protecting against mitochondria dysfunction. Aging Cell 17: e12837. [Crossref]

18. Barnes PJ, Baker J, Donnelly LE (2019) Cellular Senescence as a Mechanism and Target in Chronic Lung Diseases. Am J Respir Crit Care Med 200: 556-564. [Crossref]
19. Barnes PJ (2017) Senescence in COPD and its comorbidities. Annu Rev Physiol 79: 517-539. [Crossref]

20. Sundar IK, Rashid K, Gerloff J, Li D, Rahman I, et al. (2018) Genetic ablation of p16INK4a does not protect against cellular senescence in mouse models of chronic obstructive pulmonary disease/emphysema. Am J Respir Cell Mol Biol 59:189-199. [Crossref]

21. Cho WK, Lee CG, Kim LK (2019) COPD as a disease of Immunosenescence. Yonsei Med J 60: 407-413. [Crossref]

22. John SG, Günter S, Hager K, Conlon TM, Eickelberg O, et al. (2015) Inflammaging increases susceptibility to cigarette smoke induced COPD. Oncotarget 7: 21 . [Crossref]

Copyright: $\odot 2020$ Valério MP. This is an open-access article distributed under the terms of the Creative Commons Attribution License, which permits unrestricted use, distribution, and reproduction in any medium, provided the original author and source are credited. 